\title{
Erratum to: Effects of periodontal treatment on exacerbation frequency and lung function in patients with chronic periodontitis: study protocol of a 1-year randomized controlled trial
}

Sergio dos Santos Romero ${ }^{1}$, Erika Horácio Pinto ${ }^{2}$, Priscila Larcher Longo ${ }^{2}$, Simone Dal Corso ${ }^{3}$, Fernanda Cordoba Lanza ${ }^{3}$, Rafael Stelmach ${ }^{4}$, Samia Zahi Rached ${ }^{4}$, Adriana Lino-dos-Santos-Franco ${ }^{2}$, Marcia Pinto Alves Mayer ${ }^{5}$, Sandra Kalil Bussadori ${ }^{2,3}$, Kristianne Porta Santos Fernandes ${ }^{2,3}$, Raquel Agnelli Mesquita-Ferrari, ${ }^{2,3}$ and Anna Carolina Ratto Tempestini Horliana ${ }^{2^{*}}$

\section{Erratum}

The name of the first author was listed incorrectly in the original article [1]. The correct name of the first author is Sergio dos Santos Romero, and not Sergio Romero Santos. The correct citation of his name is "Romero SS" not "Santos RS".

\begin{abstract}
Author details
${ }^{1}$ Universidade Nove de Julho, UNINOVE, São Paulo, Brazil. ${ }^{2}$ Postgraduate program in Biophotonics Applied to Health Sciences, Universidade Nove de Julho, UNINOVE, Vergueiro, 235/249, CEP 01504-001 São Paulo, Brazil. ${ }^{3}$ Postgraduate program in Rehabilitation Sciences, Universidade Nove de Julho, UNINOVE, São Paulo, Brazil. ${ }^{4}$ Pulmonary Department, Heart Institute (InCor), School of Medicine, University of São Paulo, São Paulo, Brazil. ${ }^{5}$ Department of Microbiology, Institute of Biomedical Sciences, University of São Paulo, São Paulo, Brazil.
\end{abstract}

Received: 28 February 2017 Accepted: 10 March 2017

Published online: 14 March 2017

\section{Reference}

1. Santos RS, et al. Effects of periodontal treatment on exacerbation frequency and lung function in patients with chronic periodontitis: study protocol of a 1-year randomized controlled trial. BMC Pulm Med. 2017;17:23. doi:10.1186/ s12890-016-0340-z.

\footnotetext{
*Correspondence: annacrth@gmail.com

${ }^{2}$ Postgraduate program in Biophotonics Applied to Health Sciences,

Universidade Nove de Julho, UNINOVE, Vergueiro, 235/249, CEP 01504-001

São Paulo, Brazil
} 